\title{
(-)-Pseudodistomin E: First Asymmetric Synthesis and Absolute Configuration Assignment
}

\author{
Stephen G. Davies,* Ai M. Fletcher, Paul M. Roberts, James E. Thomson, and David Zimmer \\ Department of Chemistry, Chemistry Research Laboratory, University of Oxford, Mansfield Road, Oxford OX1 3TA, U.K. \\ Supporting Information Placeholder

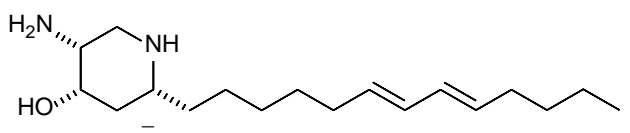 \\ ( )-Pseudodistomin E
}

\begin{abstract}
Pseudodistomin E has been prepared for the first time, allowing its structure and absolute configuration to be confirmed. The established conjugate addition of lithium ( $S$ )- $N$-allyl- $N$-( $\alpha$-methyl-p-methoxybenzyl)amide to methyl (E,E)-hepta2,5-dienoate generated the $\mathrm{C}(2)$-stereocenter, and iodolactonisation of a derivative generated the remaining two stereogenic centers. Ensuing iodide displacement was achieved using a tethering strategy, to introduce the nitrogen atom to C(5). Decarboxylative coupling of a carboxylic acid with a dialkylzinc reagent completed construction of the tridecadienyl chain.
\end{abstract}

The pseudodistomin alkaloid family comprises six members, isolated from the Okinawan tunicate Pseudodistoma kanoko by Kobayashi et al. in 1987 (pseudodistomins A and B) ${ }^{1}$ and 1995 (pseudodistomin C), ${ }^{2}$ and from the ascidian Pseudodistoma megalarva by Freyer et al. in 1997 (pseudodistomins $\mathrm{D}, \mathrm{E}$ and F). ${ }^{3,4}$ Together, they represent one of the rare examples of the isolation of alkaloids from sea creatures. Unsurprisingly, there has been interest in the development of syntheses of members of this family of alkaloids in both racemic and enantiopure form, with routes to pseudodistomins $\mathrm{A},{ }^{5} \mathrm{~B},{ }^{5-9}$ $\mathrm{C},{ }^{10-12} \mathrm{D},{ }^{13,14}$ and $\mathrm{F}^{7}$ having been reported to date. These endeavors have enabled not only the structures of the alkaloids to be confirmed (in fact, the structures of pseudodistomins A and B originally proposed by Kobayashi et $a l .{ }^{15}$ were revealed to be erroneous and were thus revised), ${ }^{6,16,17}$ but also their absolute configurations. It is uniquely pseudodistomin $\mathrm{E}$ that remains to acquiesce to laboratory synthesis, and hence receive confirmation of its assigned structure and absolute configuration. The gross structure of pseudodistomin E was assigned by Freyer et al. using a combination of evidence, mainly gathered through NMR spectroscopic investigations. ${ }^{3}{ }^{1} \mathrm{H}$ NMR ${ }^{3} J$ coupling constant analysis was used to assign the $(E, E)$ configuration to the diene unit of the side chain, as well as the relative configurations of the three stereogenic centers around the piperidine ring. ${ }^{3}$ This analysis indicated that pseudodistomin $\mathrm{E}$ possessed the same relative configuration of the three stereogenic centers as pseudodistomin C. As the magnitudes of the specific rotation values of pseudodistomin $\mathrm{E}$ $\left\{[\alpha]_{\mathrm{D}}{ }^{25}-20.8\right.$ (c 0.39 in $\left.\left.\mathrm{MeOH}\right)\right\}$ and pseudodistomin $\mathrm{C}$ $\left\{[\alpha]_{\mathrm{D}}{ }^{24}-24(c \quad 0.7 \text { in } \mathrm{MeOH})\right\}^{18}$ were similar and their signs the same, and given that their structures differed only in the identity of the unsaturated hydrocarbon chain at C(2), Freyer et al. reasoned that the two natural products would be of the same enantiomeric form. As pseudodistomin C had already been shown to possess the $(2 S, 4 S, 5 R)$ absolute configuration by both degradation studies ${ }^{2}$ and total synthesis from D- serine,${ }^{10}$ Freyer et al. assigned the $(2 R, 4 S, 5 R)$ absolute configuration to pseudodistomin $E^{3}{ }^{3}$ Herein, we report the first asymmetric synthesis of pseudodistomin E, which both confirms its structure and enables the $(2 R, 4 S, 5 R)$ absolute configuration of the three stereogenic centers of the piperidine ring to be assigned unambiguously.

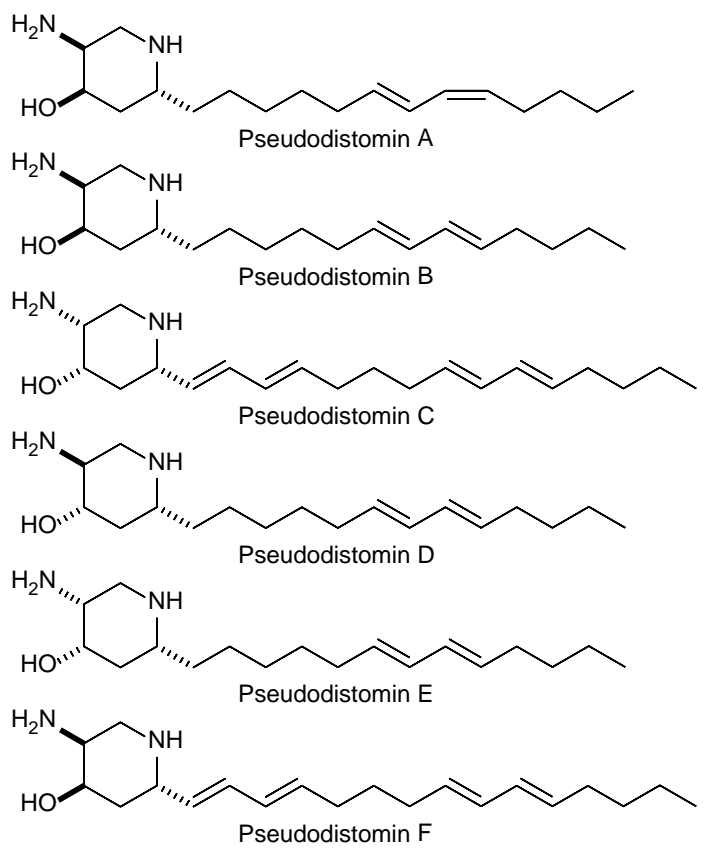

Figure 1. Structures of the pseudodistomin alkaloids.

Our asymmetric synthesis of pseudodistomin $\mathrm{D}^{14}$ used the diastereoselective conjugate addition of lithium (S)- $N$-allyl- $N$ ( $\alpha$-methyl-p-methoxybenzyl)amide ${ }^{19}$ to methyl $(E, E)$-hepta2,5-dienoate $\mathbf{1}$ as a stereodefining step in the preparation of lactone 7. Elaboration of $\mathbf{7}$ to pseudodistomin D involved for- 
mal substitution of the iodide functionality by an amino functionality with overall retention of configuration. ${ }^{14}$ We therefore envisaged elaboration of 7 to pseudodistomin $\mathrm{E}$ via formal substitution of the iodide functionality by an amino functionality with overall inversion of configuration; this obviously suggested an $\mathrm{S}_{\mathrm{N}} 2$-type reaction. Lactone 7 was prepared as previously reported, ${ }^{14}$ in six steps and $75 \%$ overall yield from methyl (E,E)-hepta-2,5-dienoate. Attempted intermolecular displacement of the iodide functionality within 7 by a range of nucleophilic nitrogen sources $\left(\mathrm{LiN}_{3}, \mathrm{NaN}_{3}, \mathrm{PhthNK}, \mathrm{BnNH}_{2}\right.$, $\mathrm{Bn}_{2} \mathrm{NH}$ ) was, however, thwarted by either no reaction or the formation of $N$-Boc enamine $\mathbf{8}$, with none of the corresponding substitution product $\mathbf{9}$ being observed under any of the conditions investigated. This is consistent with an E2-type elimination dominating, which is rendered a facile process by the favorable antiperiplanar arrangement of the $\mathrm{C}(8)$-iodide functionality with one of the vicinal $\mathrm{C}(7)$-protons on the bicyclic framework of $7 .^{20}$ In fact, treatment of 7 with DBU provided 8 in $87 \%$ yield (Scheme 1).

\section{Scheme 1.}

$$
\text { (1, 90\% purity }
$$

PMP $=p$-methoxyphenyl. PhthNK $=$ potassium phthalimide.

An alternative approach based upon tethered (i.e., intramolecular) delivery of the nucleophilic nitrogen atom was next explored. ${ }^{21}$ Reduction of lactone 7 with DIBAL-H gave the corresponding lactol 10, which existed mainly ( 90\%) as the open-chain form $\mathbf{1 1}^{14}$ according to ${ }^{1} \mathrm{H}$ NMR spectroscopic analysis in $\mathrm{CDCl}_{3}$. Treatment of $\mathbf{1 1}$ with TsNCO gave a 5:88:7 mixture of unreacted 11, $\mathrm{N}$-Ts carbamate 12 and vinyl ether 14, along with $\mathrm{TsNH}_{2}$. Stirring this mixture with $\mathrm{Et}_{3} \mathrm{~N}$ in acetone at $60{ }^{\circ} \mathrm{C}$ resulted in formation of a 10:81:9 mixture of unreacted 11, $N$-Ts oxazolidinone 13, and vinyl ether 14, along with $\mathrm{TsNH}_{2}$. Purification gave 11 in 10\% yield ( 95\% purity), 13 in 63\% yield (contaminated with $\sim 15 \% \mathrm{TsNH}_{2}$ ), and 14 in $4 \%$ yield. These product distributions suggest that a competing dehydration process is occurring: reaction of the hydroxyl functionality within the closed, lactol form 10 (rather than open-chain form 11) with TsNCO followed by fragmentation gives 14 and $N$-tosylcarbamic acid, and decarboxylation of the latter gives $\mathrm{TsNH}_{2}{ }^{22}$ Unfortunately, treatment of $\mathbf{1 3}$ with $\mathrm{Ph}_{3} \mathrm{P}=\mathrm{CH}\left(\mathrm{CH}_{2}\right)_{3} \mathrm{OBn}$ [to subsequently facilitate $\mathrm{C}\left(2^{\prime}\right)$ side-chain construction in an analogous fashion to that of our pseudodistomin D synthesis $]^{14}$ delivered a complex mixture of products from which 15 was isolated in 33\% yield ( 95\% purity) and $>95: 5 \mathrm{dr}[(Z):(E)$ ratio]; the corresponding product 16 in which the oxazolidinone ring had been cleaved was isolated as the only other identifiable species in 3\% yield ( $95 \%$ purity) and $>95: 5 \mathrm{dr}[(Z):(E)$ ratio]. The geometries of the newly formed olefins were assigned from the diagnostic values of the ${ }^{1} \mathrm{H}$ NMR ${ }^{3} J$ coupling constants, with ${ }^{3} J<11 \mathrm{~Hz}$ in both cases (Scheme 2).

\section{Scheme 2 .}

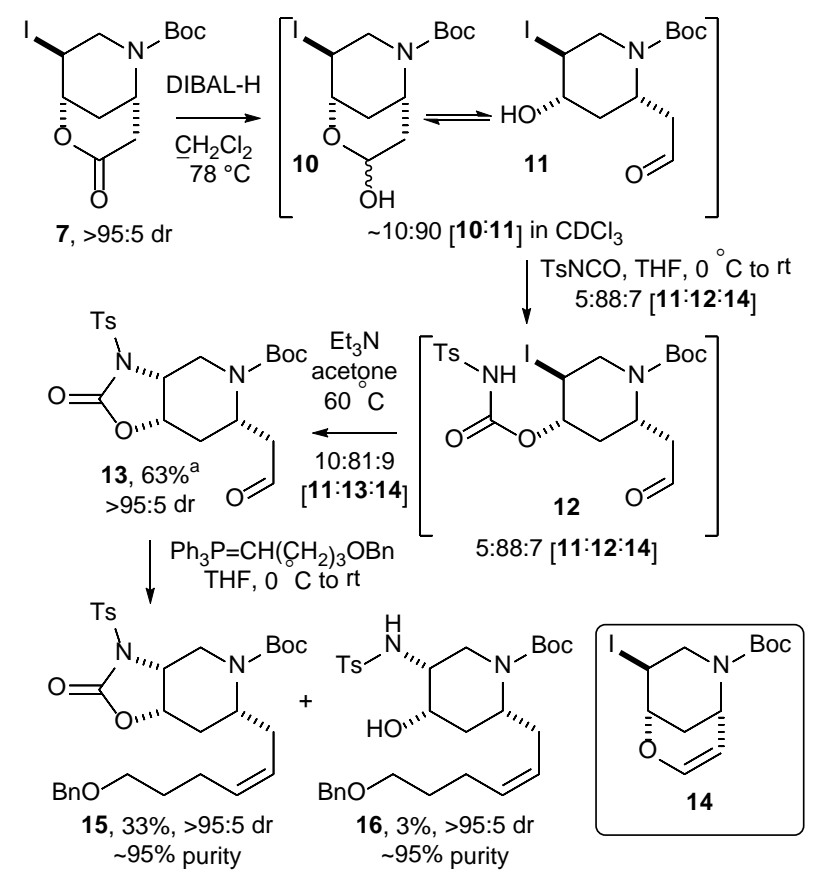

${ }^{a}$ Contaminated with $~ 15 \% \mathrm{TsNH}_{2}$.

In order to eradicate the competing dehydration pathway (and, hence, formation of $\mathrm{TsNH}_{2}$ ), the possibility of effecting olefination of the aldehyde functionality prior to isocyanate trapping and cyclic carbamate formation was considered. Although reaction of 11 with $\mathrm{Ph}_{3} \mathrm{P}=\mathrm{CH}\left(\mathrm{CH}_{2}\right)_{3} \mathrm{OBn}$ has already been shown to result in olefination and epoxide formation (undesired in this case), ${ }^{14}$ reaction of 11 with $\mathrm{Ph}_{3} \mathrm{P}=\mathrm{CHCO}_{2} \mathrm{Me}$ resulted in olefination only, with 17 being isolated in $90 \%$ yield (from 7 ) and 68:32 dr [(E):(Z) ratio]. Subsequent treatment of 17 with TsNCO gave $N$-Ts carbamate 18 in 68:32 dr [(E):(Z) ratio], and then exposure of 18 to $\mathrm{Et}_{3} \mathrm{~N}$ gave $N$-tosyl oxazolidinone 19 in 91\% yield (from 17) and 68:32 dr [(E):(Z) ratio]. Hydrogenation of $\mathbf{1 9}$ gave 20 in $>95: 5 \mathrm{dr}$, thus establishing conclusively that $68: 32 \mathrm{dr}$ corresponds to the ratio of geometric olefin isomers for 17-19. Methanolysis of the carbamate functionality within 20 using $\mathrm{K}_{2} \mathrm{CO}_{3}$ in $\mathrm{MeOH}$ gave 21 in $77 \%$ yield (from 19). The relative configuration of 21 was then assigned from ${ }^{1} \mathrm{H}$ NMR ${ }^{3} J$ coupling constant analysis, assuming a chair conformation. This not only established the relative configurations within 19 and 20 but also that the formal substitution of the iodide functionality by an amino func- 
tionality had proceeded with inversion of configuration, as required for the synthesis of pseudodistomin E. With this transformation (i.e., 7 to 19) established, an end-game for the elaboration of lactone 7 into pseudodistomin $\mathrm{E}$ using the decarboxylative coupling of a carboxylic acid and a dialkylzinc reagent, recently reported by Baran et al., ${ }^{23}$ was envisaged. Thus, sequential treatment of lactone 7 with DIBAL-H, $\mathrm{Ph}_{3} \mathrm{P}=\mathrm{CHCO}_{2} \mathrm{Bn}$, TsNCO and $\mathrm{Et}_{3} \mathrm{~N}$ gave $N$-Ts oxazolidinone $\mathbf{2 4}$ (the analogue of $\mathbf{1 9}$ with a pendant benzyl rather than methyl ester) in 77\% overall yield and 68:32 dr [(E):(Z) ratio]. Tandem hydrogenation/hydrogenolysis of $\mathbf{2 4}$ gave carboxylic acid 25 which was coupled with PhthNOH to give activated ester 26 in 96\% yield and >95:5 dr (Scheme 3). The coupling partner, (E,E)-1-bromodeca-3,5-diene 29, was prepared via 1,2-addition of cyclopropylmagnesium bromide to (E)-2heptenal 27 ( $>95: 5 \mathrm{dr}$ ) and then rearrangement/substitution of the resultant secondary alcohol 28 promoted by $48 \%$ aq $\mathrm{HBr}$ for $24 \mathrm{~h},{ }^{24}$ giving 29 in 86:7:7 dr [( $\left.E, E\right):(3 E, 5 Z):(3 Z, 5 E)$ ratio] and $91 \%$ isolated yield (Scheme 4$)$.

\section{Scheme 3.}

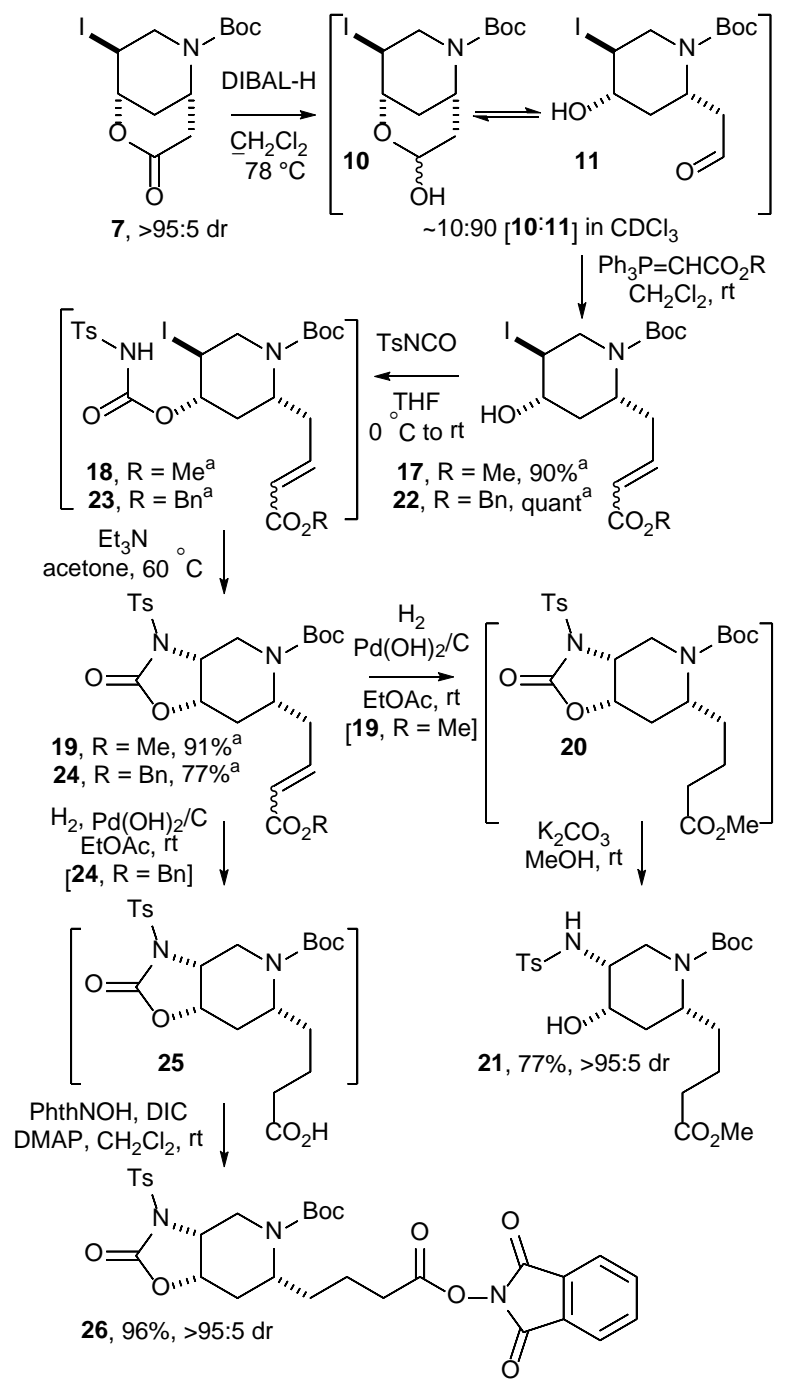

${ }^{a} 68.32 \mathrm{dr}[(E):(Z)$ ratio]. PhthNOH $=N$-hydroxyphthalimide. DIC $=N, N^{\prime}$-diisopropylcarbodiimide.

Following Baran's protocol, ${ }^{23}$ ester 26 was coupled with bromide $\mathbf{2 9}$ to give $\mathbf{3 0}$ in 52\% yield and 82:8:8:2 dr [(E,E):(6'E, $\left.8^{\prime} Z\right):\left(6^{\prime} Z, 8^{\prime} E\right):(Z, Z)$ ratio]. The final stages involved
$N$ - and $O$-deprotection: the $N$-Ts group was removed from 30 upon treatment with sodium naphthalenide to give 31 in $89 \%$ yield and 82:8:8:2 dr [(E,E):(6'E,8'Z):(6'Z,8'E):(Z,Z) ratio], the oxazolidinone moiety within $\mathbf{3 1}$ was cleaved using $\mathrm{KOH}$ in hot $\mathrm{MeOH}$ to give 32, and finally the $N$-Boc group was removed from 32 using $\mathrm{HCl}$ in hot $\mathrm{MeOH}$ to give synthetic pseudodistomin E 33 in 72\% yield (from 31) and 82:8:8:2 dr $\left[(E, E):\left(6^{\prime} E, 8^{\prime} Z\right):\left(6^{\prime} Z, 8^{\prime} E\right):(Z, Z)\right.$ ratio]. The integrity of the diene geometry is clearly somewhat compromised during the coupling reaction, but the deprotection steps occur without further isomerization. As the lithium amide reagent used for the conjugate addition reaction was $>99 \%$ ee, 33 was inferred as being $>99 \%$ ee, along with all intermediates en route (Scheme 5).

Scheme 4.

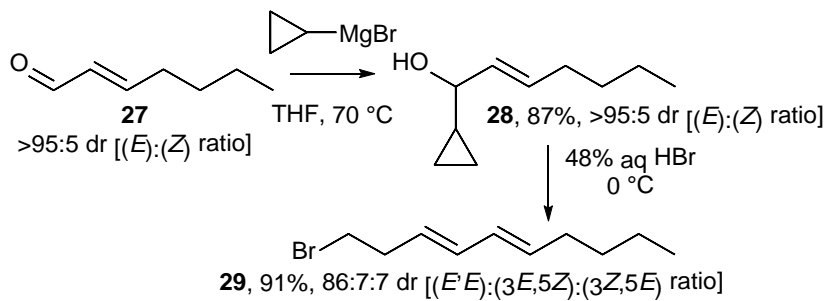

Scheme 5.
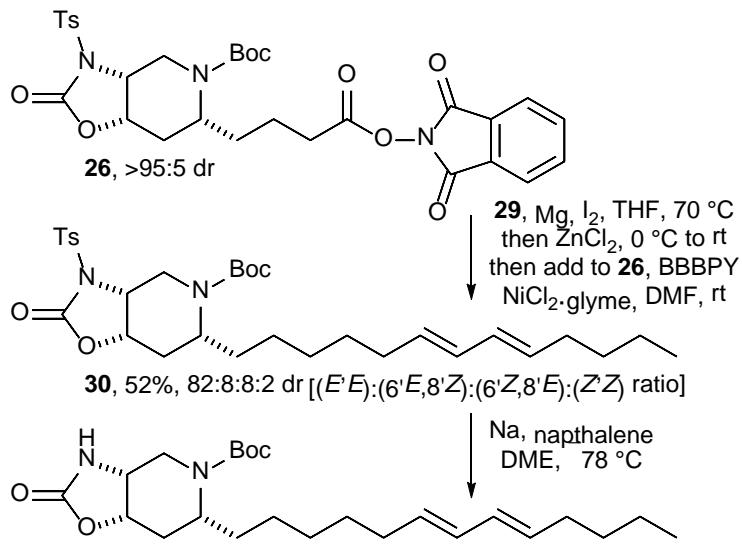

31, 89\%, 82:8:8:2 dr [( $\left.E^{\prime} E\right):\left(6^{\prime} E, 8^{\prime} Z\right):\left(6^{\prime} Z, 8^{\prime} E\right):\left(Z^{\prime} Z\right)$ ratio]

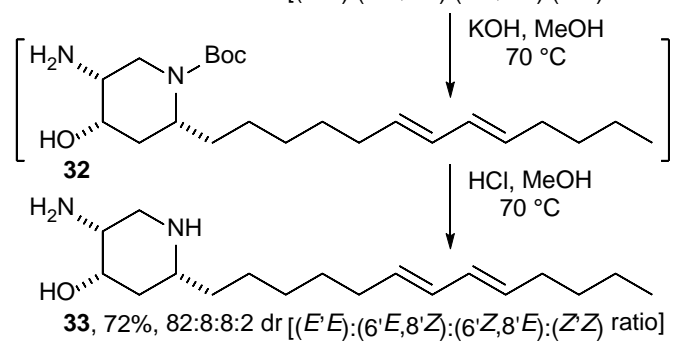

BBBPY = 4,4'-di-tert-butyl-2,2'-bipyridine.

${ }^{1} \mathrm{H}$ NMR ${ }^{3} J$ coupling constant analysis of $\mathbf{3 3}$ was evincive of the relative configuration around the piperidine ring [a chair conformation with the $\mathrm{C}(2)$ - and $\mathrm{C}(4)$-substituents equatorial, and the $\mathrm{C}(5)$-substituent axial], as well as the (E,E)-geometry of the diene unit. ${ }^{25}$ As may be reasonably expected for a compound containing two basic amino functionalities, the ${ }^{1} \mathrm{H}$ and ${ }^{13} \mathrm{C}$ NMR spectroscopic data of $\mathbf{3} 3$ showed significant variance upon introduction of trifluoroacetic acid (in 0.1 equiv portions) to the sample (Figure 2 and Supporting Information). A similar effect on the magnitude of the specific rotation value of 33 was also noted: for example we obtained $[\alpha]_{D}{ }^{25}-34.5(c$ 
1.0 in $\mathrm{MeOH}$ ) and $[\alpha]_{\mathrm{D}}{ }^{25}-9.2$ (c 1.0 in $1.25 \mathrm{M}$ methanolic $\mathrm{HCl}$ ). Comparison of our spectroscopic and physical data for 33 with those reported by Freyer et al. for pseudodistomin $\mathrm{E}^{3}$ revealed excellent agreement of the ${ }^{1} \mathrm{H}$ NMR spectroscopic data of 33 (free base) with those of the natural product $\left(\Delta \delta_{\mathrm{H}} \leq\right.$ $0.02 \mathrm{ppm}) .{ }^{26}$ Meanwhile, the ${ }^{13} \mathrm{C}$ NMR spectroscopic data of 33-0.1TFA showed excellent parity with those reported for the natural product $\left(\Delta \delta_{\mathrm{C}} \leq 0.2 \mathrm{ppm}\right),{ }^{26}$ suggesting that traces of acid were present in the sample of the natural product when the ${ }^{13} \mathrm{C}$ NMR spectrum was recorded. The specific rotation of 33.0.1TFA was measured as $[\alpha]_{\mathrm{D}}{ }^{25}-22.0$ (c 0.5 in $\left.\mathrm{MeOH}\right)$, which compares favorably with $[\alpha]_{\mathrm{D}}{ }^{25}-20.8$ (c 0.39 in $\mathrm{MeOH})\}$ reported by Freyer et al. for pseudodistomin E. ${ }^{3}$ It can thus be concluded that $\mathbf{3} 3$ and pseudodistomin $\mathrm{E}$ are identical, hence establishing unambiguously the structure and $(2 R, 4 S, 5 R)$ absolute configuration of the natural product.

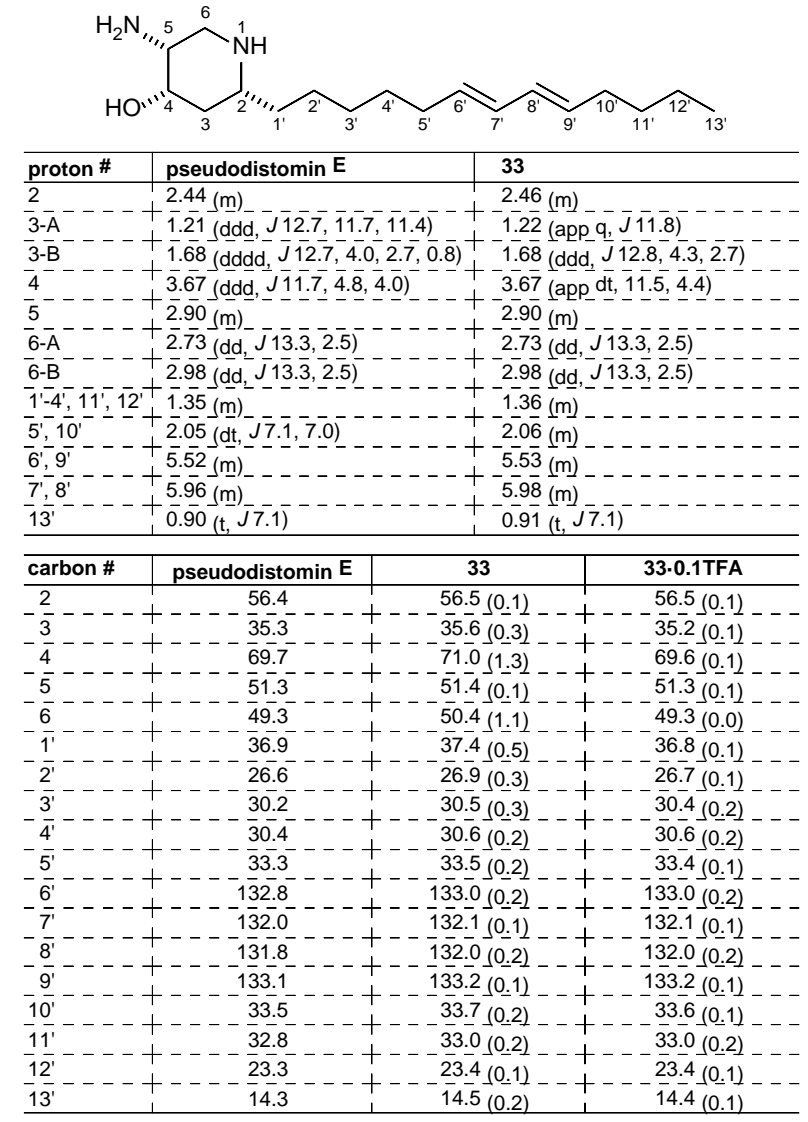

Figure 2. ${ }^{1} \mathrm{H}(400 \mathrm{MHz})$ and ${ }^{13} \mathrm{C}(100 \mathrm{MHz}) \mathrm{NMR}$ spectroscopic data for pseudodistomin $\mathrm{E}$ and synthetic 33 in $d_{4}-\mathrm{MeOH}$. Values of $\Delta \delta \mathrm{C}$ are given in parentheses.

In conclusion, the first asymmetric synthesis of (-)pseudodistomin $E$ has been achieved in sixteen steps from methyl (E,E)-hepta-2,5-dienoate, and in 19\% overall yield and $>99 \%$ ee. Conjugate addition of lithium (S)- $N$-allyl- $N-(\alpha-$ methyl-p-methoxybenzyl)amide to methyl (E,E)-hepta-2,5dienoate generated the $C(2)$-stereocenter of the target, with regioselective iodolactonisation of a derivative constructed the remaining $\mathrm{C}(4)$ - and $\mathrm{C}(5)$-stereocenters. Iodide displacement (with inversion of configuration) was achieved using a tethering strategy, and set the absolute C(5)-stereochemistry required for the target. The unsaturated C(2)-hydrocarbon chain was constructed using a decarboxylative coupling of a carboxylic acid with a dialkylzinc reagent. Comparison of NMR spectroscopic and specific rotation data of our synthetic sample with those of the natural product established conclusively both the structure and $(2 R, 4 S, 5 R)$ absolute configuration of pseudodistomin E.

\section{ASSOCIATED CONTENT}

\section{Supporting Information}

The Supporting Information is available free of charge on the ACS Publications website at DOI: XXXX.

Experimental details, characterization data, and copies of ${ }^{1} \mathrm{H}$ and ${ }^{13} \mathrm{C}$ NMR spectra (PDF)

\section{AUTHOR INFORMATION}

\section{Corresponding Author}

*E-mail: steve.davies@chem.ox.ac.uk.

\section{Notes}

The authors declare no competing financial interest.

\section{REFERENCES}

(1) Ishibashi, M.; Ohizumi, Y.; Sasaki, T.; Nakamura, H.; Hirata, Y.; Kobayashi, J. J. Org. Chem. 1987, 52, 450.

(2) Kobayashi, J.; Naitoh, K.; Doi, Y.; Deki, K.; Ishibashi, M. J. Org. Chem. 1995, 60, 6941.

(3) Freyer, A. J.; Patil, A. D.; Killmer, L.; Troupe, N.; Mentzer, M.; Carte, B.; Faucetter, L.; Johnson, R. K. J. Nat. Prod. 1997, 60, 986.

(4) Freyer et al. also isolated the known pseudodistomins B and C alongside the new pseudodistomins D-F (see ref 3).

(5) Kiguchi, T.; Yuumoto, Y.; Ninomiya, I.; Naito, T. Chem. Pharm. Bull. 1997, 45, 1212.

(6) Kiguchi, T.; Yuumoto, Y.; Ninomiya, I.; Naito, T.; Deki, K.; Ishibashi, M.; Kobayashi, J. Tetrahedron Lett. 1992, 33, 7389.

(7) Ma, D.; Sun, H. J. Org. Chem. 2000, 65, 6009.

(8) Davis, F. A.; Zhang, J.; Li, Y.; Xu, H.; DeBrosse, C. J. Org. Chem. 2005, 70, 5413 .

(9) Lee, J. H.; Jeong, W.; Rhee, Y. H. Synthesis 2014, 46, 2155.

(10) Doi, Y.; Ishibashi, M.; Kobayashi, J. Tetrahedron 1996, 52, 4573.

(11) Langlois, N. Org. Lett. 2002, 4, 185.

(12) Tanaka, K.; Maesoba, T.; Sawanishi, H. Heterocyles 2006, 68, 183.

(13) Trost, B. M.; Fandrick, D. R. Org. Lett. 2005, 7, 823.

(14) Davies, S. G.; Fletcher, A. M.; Lee, J. A.; Roberts, P. M.; Russell, A. J.; Taylor, R. J.; Thomson, A. D.; Thomson, J. E. Org. Lett. 2012, 14, 1672.

(15) Pseudodistomin A was originally assigned as (2R,4R,5S,3'E, 5'Z)-2-(trideca-3',5'-dien-1'-yl)-5-aminopiperidin-4-ol and pseudodistomin $\mathrm{B}$ was originally assigned as $(2 R, 4 R, 5 S, E, E)-2-$ (trideca-3',5'-dien-1'-yl)-5-aminopiperidin-4-ol (see ref 1).

(16) Naito, T.; Yuumoto, Y.; Kiguchi, T.; Ninomiya, I. J. Chem. Soc., Perkin Trans. 1 1996, 281.

(17) Ishibashi, M.; Deki, K.; Kobayashi, J. J. Nat. Prod. 1995, 58, 804.

(18) No specific rotation value for pseudodistomin C was reported in either of the two publications detailing its isolation (see ref 2 and ref 3); the value quoted is for the synthetic sample prepared by Kobayashi et al. (see ref 10).

(19) For development of lithium $N$-allyl- $N$-( $\alpha$-methylbenzyl)amide, see: Davies, S.G.; Fenwick, D.R.; Ichihara, O. Tetrahedron: Asymmetry 1997, 8, 3387.

(20) The solid state conformation of 7 (see ref 14) displays this geometric arrangement. 
(21) For examples of this tactic, see: (a) Knapp, S.; Kukkola, P. J.; Sharma, S.; Pietranico, S. Tetrahedron Lett. 1987, 28, 5399. (b) Das, J. Synth. Commun. 1988, 18, 907.

(22) The ratio of vinyl ether $\mathbf{1 4}$ to $\mathrm{TsNH}_{2}$ was $\sim 1: 1$ in the ${ }^{1} \mathrm{H}$ NMR spectrum of both of the relevant crude reaction mixtures.

(23) Qin, T.; Cornella, J.; Li, C.; Malins, L. R.; Edwards, J. T.; Kawamura, S.; Maxwell, B. D.; Eastgate, M. D.; Baran, P. S. Science 2016, 352, 801.

(24) Svirskaya, P. I.; Maiti, S. N.; Jones, A. J.; Khouw, B.; Leznoff, C. C. J. Chem. Ecol. 1984, 10, 795.

(25) ${ }^{3} J_{6^{\prime}, 7^{\prime}}$ and ${ }^{3} J_{8^{\prime}, 9^{\prime}}$ were discerned as $>15 \mathrm{~Hz}$.

(26) $\Delta \delta \mathrm{x}=\mid \delta \mathrm{x}$ (synthetic) $-\delta \mathrm{x}$ (natural) $\mid$, where $\mathrm{X}=\mathrm{H}$ or $\mathrm{C}$. 